Published in final edited form as:

J Comp Neurol. 2009 April 10; 513(5): 441-456. doi:10.1002/cne.21993.

\title{
Color processing in the medulla of the bumblebee (Apidae: Bombus impatiens)
}

\author{
Angelique C. Paulk ${ }^{1}$, Andrew M. Dacks ${ }^{2}$, Wulfila Gronenberg ${ }^{2}$ \\ 1.Queensland Brain Institute, Bldg 79, The University of Queensland, St. Lucia, QLD 4072, \\ Australia \\ 2.ARL Division of Neurobiology, University of Arizona, Tucson, AZ 85721, U. S. A.
}

\begin{abstract}
The mechanisms of processing a visual scene involve segregating features (such as color) into separate information channels at different stages within the brain, processing these features, and then integrating this information at higher levels in the brain. To examine how this process takes place in the insect brain, we focused on the medulla, an area within the optic lobe through which all of the visual information from the retina must pass before it proceeds to central brain areas. We used histological and immunocytochemical techniques to examine the bumblebee medulla and found that the medulla is divided into eight layers. We then recorded and morphologically identified 27 neurons with processes in the medulla. During our recordings, we presented color cues to determine whether response types correlated with locations of the neural branching patterns of the filled neurons among the medulla layers. Neurons in the outer medulla layers had less complex color responses compared to neurons in the inner medulla layers and there were differences in the temporal dynamics of the responses among the layers. Progressing from the outer to the inner medulla, neurons in the different layers appear to process increasingly complex aspects of the natural visual scene.
\end{abstract}

\section{Graphical Abstract}

\section{Keywords}

insect brain; temporal response properties; adaptation

\section{INTRODUCTION}

Processing a visual scene involves a complex series of physiological transformations that occur at successive stages within the brain. In the vertebrate visual system, information is sequentially processed within the retinal network from which parallel streams of information supply higher-order visual processing centers, such as the visual cortex, in which different features of visual stimuli are extracted by functional subsets of neurons (Callaway, 2005;

Corresponding author Angelique C. Paulk, Queensland Brain Institute, Bldg 79, The University of Queensland, St. Lucia, QLD 4072, Australia, Phone: +61 414665 478, Fax: +61 73346 6301, a.paulk@uq.edu.au. 
Hubel and Livingstone, 1987). Insects appear to rely on the same basic design of visual processing to extract increasingly complex features from the original photoreceptor signals (Strausfeld, 1989). As in vertebrates, visual information originating from insect photoreceptors is transformed through a series of processing stages until the information is integrated in the central brain.

A crucial stage in the insect visual processing pathway is the medulla. It is the second and largest neuropil in the insect brain's optic lobe, containing the largest number of neurons (Strausfeld, 1976; Witthöft, 1967) and neural types of the optic neuropil (Ribi and Scheel, 1981; Strausfeld, 1976). Visual signals are detected by photoreceptors in the retina, which input to the lamina (the first visual neuropil), which then inputs to the medulla. The inputs and the overall structure of the medulla is retinotopic, with a point-to-point representation of the eye and the external world (Ribi and Scheel, 1981; Strausfeld, 1976). This retinotopic design results in a columnar organization, where each point in space is processed by a distinct assemblage of neurons referred to as 'cartridges' in the lamina and 'columns' in the medulla (Strausfeld, 1976).

Unlike vertebrates, the insect retina comprises only photoreceptors and no interneurons. The lamina receives the axons of specific types of retinal photoreceptors and is composed mainly of many small field columnar neurons arranged in a lattice array where each cartridge matches the photoreceptor input (Campos-Ortega and Strausfeld, 1972; Ribi, 1975a, 1975b). In contrast, the medulla is composed of columnar elements as well as a number of horizontal elements crossing the columnar units and interconnecting them laterally, indicating that the medulla contains many processing elements and stages within the structure itself. The medulla itself is layered in the distal - proximal direction (perpendicular to the long axis of the columns), with differing numbers of columnar and horizontal neurons dividing the structure into additional processing stages (Ribi and Scheel, 1981; Strausfeld, 1976). In both bees and flies, a great diversity of neurons occupies the medulla stratifications. The branching patterns of some neurons are restricted to single layers while others branch extensively throughout the medulla (Fischbach and Dittrich, 1989; Ribi and Scheel, 1981). Different kinds of intrinsic elements connect the layers, while extrinsic neurons project from the medulla layers to other brain regions. Together, they form parallel visual pathways through the medulla (Fischbach and Dittrich, 1989; Douglass and Strausfeld, 1996; Ribi and Scheel, 1981). Color processing requires the comparison of signals between the short, middle, and long wavelength type photoreceptors and several conjectures exist as to the location and neuronal composition of the color processing pathway within and beyond the lamina and medulla (Morante and Desplan, 2008).

The fact that lamina amacrine neurons connect different cartridges of lamina neurons and spines along laminar monopolar neurons suggests that there is communication across color channels within lamina cartridges (Fischbach and Dittrich, 1989; Douglass and Strausfeld, 2005; Souza et al., 1987). This suggests that initial steps of color processing occur in the lamina and are communicated to the medulla (Menzel, 1974; de Souza, 1992), although the axons of short-wavelength photoreceptors (blue to ultraviolet; R7 - R9 in bees) proceed through the lamina, apparently without contacting laminar neurons, and terminate in the medulla. This is in contrast to the majority of photoreceptors (R1-R6), which process longer 
wavelengths and terminate in the lamina (Campos-Ortega and Strausfeld, 1972; Gribakin, 1969, 1972; Morante and Desplan, 2008; Ribi, 1975a). This suggests that the medulla is the first stage in the visual pathway where full color information can be extracted by comparison across different receptor 'channels' to be sent to higher-order centers of the brain.

Most medulla neurons project to the third optic neuropil, the lobula complex. However, in addition, a substantial part of medulla elements output directly in the central brain, circumventing the lobula complex (Fischbach and Dittrich, 1989; Ribi and Scheel, 1981; Strausfeld, 1976). Hence, the medulla provides output to the lobula complex as well as directly to the central brain, bypassing the lobula complex, potentially carrying different streams of information or using different neural encoding strategies.

Despite the obvious anatomical significance of the medulla, there have been relatively few studies on the physiological properties of medulla neurons in insects. Neurons in the medulla have demonstrated an enormous variety of responses, as one would expect in view of the large number of neuronal types. One would therefore predict that because most of the visual information relayed to the central brain must pass through the medulla, the medulla likely processes many visual sub-modalities. Accordingly, neurons in the medulla have been shown to be color sensitive and color opponent (Hertel, 1980; Hertel et al., 1987; Hertel and Maronde, 1987), motion sensitive (Douglass and Strausfeld, 2003, 2007; Gilbert et al., 1991; O'Carroll et al., 1992; Osorio, 1986), polarization sensitive (Homberg and Würden, 1997), and to have spatially antagonistic receptive fields (Hertel, 1980). However, considering the relatively few recordings made in the medulla, a structure containing nearly 147,500 neurons in the fly (Strausfeld, 1976), we have yet to fully characterize the distribution of physiological response properties among neurons within the medulla layers, particularly in terms of color processing.

Since the medulla may form the initial stage of color processing in the bee brain due to the distribution of photoreceptor inputs, the layers could represent progressive stages of color processing. As spectral information moves through the layers of the medulla, neurons could produce increasingly complex responses which could include color opponent responses or antagonistic receptive fields. Therefore, we wanted to examine whether neurons belonging to the medulla layers could be exhibiting differences in their responses to color.

To do so, we recorded from medulla neurons in bumblebees (Bombus impatiens) intracellularly while presenting visual cues and filling the neurons with fluorescent dye to determine their projection patterns in the medulla. Bumblebees are highly visual insects, with advanced visual learning capabilities (Ney-Nifle et al., 2001) and trichromatic color vision (Peitsch et al., 1992). They are closely related to honeybees (Roig-Alsina et al., 1993; Schultz et al., 2001), which have been particularly well-studied insect models regarding visual behavior (Giurfa, 2007; Menzel and Giurfa, 2006; Srinivasan, 1993; Srinivasan and Zhang, 1998). To better understand the neural basis of this capacity for visual learning, we sought to characterize the physiological response properties of medulla neurons of the bumblebee (Bombus impatiens) to color stimuli. 


\section{MATERIALS AND METHODS}

\section{Animals}

Bumblebee colonies (Bombus impatiens) were acquired from Biobest Biological Systems (International Technology Services, MI) and were maintained in the laboratory. We selected bumblebees of unknown age for electrophysiological recordings $(n=26)$, mass fills of neurons ( $\mathrm{n}=3$ ), Bodian staining $(\mathrm{n}=6)$, and immunocytochemistry $(\mathrm{n}=18)$.

\section{Anatomy}

To examine output connections within the medulla, we used anterograde tract tracing as described by Ehmer and Gronenberg (2002). Briefly, crystals of Texas red ${ }^{\circledR}$ or fluorescein conjugated to $3000 \mathrm{MW}$ dextran (Molecular Probes, Invitrogen) were placed on the tips of fine-tipped capillary tubes which were used to penetrate cell bodies near the medulla or in the retina to fill neurons. The area was sealed with wax and the bee was kept at $4{ }^{\circ} \mathrm{C}$ overnight. The next day, the brain tissue was processed as described below.

\section{Histology}

Bee brains with fluorescent intracellular or mass fill labeling were fixed (4\% paraformaldehyde in buffered insect saline, $\mathrm{pH}$ 6.9), dissected, rinsed, and dehydrated using increasing concentrations of ethanol and embedded in Spurr's low viscosity medium (EMS, Hartfield, PA) and polymerized. The resulting plastic blocks were sectioned at 10-20 $\mu \mathrm{m}$ using a sliding microtome, mounted and cover-slipped. Images of the brain sections were recorded using a Nikon PCM 2000 confocal laser microscope with argon and HeNe lasers (Nikon, Japan) or a Zeiss Pascal confocal attached to a Zeiss Axioplan microscope. Neurons were reconstructed from the image stacks and were adjusted for brightness and contrast using Photoshop (Adobe, San Jose, CA). In all figures including neurons, unless otherwise indicated, the images are two-dimensional projections and reconstructions across all plastic sections where the neurons were present. Three-dimensional reconstructions were performed using a custom Matlab (Natick, MA) program.

To examine the brain anatomy and neuronal pathways, we used Bodian reduced silver staining similar to the protocol described by Gregory (1980). Brains were removed from the head capsule and fixed overnight at $4^{\circ} \mathrm{C}$ in A.A.F. (64\% Ethyl-Alcohol, 5\% Acetic Acid, and $3.75 \%$ Formalin). After fixation the tissue was dehydrated and embedded in paraffin (Paraplast+, Oxford Labware, St. Louis, MO). Paraffin blocks were cut into $15 \mu \mathrm{m}$ sections mounted on slides coated with albumin, deparaffinized and rehydrated before incubation in protargol solution: $2.5 \mathrm{~g}$ silver protein (Alfa Aeser) and $2 \mathrm{~g}$ metallic copper, in $250 \mathrm{ml}$ distilled water. After 23 hours at $60^{\circ} \mathrm{C}$ slides were removed from the protargol solution and processed through $1 \%$ hydroquinone and $5 \%$ sodium sulphite for $5 \mathrm{~min}, 1 \%$ gold chloride under bright light for $7 \mathrm{~min}$, (1\%) oxalic acid for $5 \mathrm{~min}$ and $2.5 \%$ sodium thiosulfate for 5 min. Sections were again dehydrated and then cover-slipped using Entellan (Merck). Finally, we performed Golgi impregnations of neurons using the methods described by Ribi and Scheel (1981). 


\section{Immunohistochemistry}

Tissue from an additional eighteen bumblebees was labeled for serotonin (5HT; $\mathrm{n}=6$ ), histamine ( $\mathrm{n}=6)$, and GABA $(\mathrm{n}=6)$ immunoreactivity using the protocol modified from Dacks et al. (2006) and Homberg and Hildebrand (1991). Briefly, brains were dissected and then fixed overnight in $4 \%$ paraformaldehyde at $4^{\circ} \mathrm{C}$, except for the histamine staining in which brains were prefixed for 4 hours in 1-ethyl-3-(3-dimethylaminopropyl) carbodiimide (Sigma, St. Louis, MO) in double de-ionized water at $4{ }^{\circ} \mathrm{C}$ and then placed in $4 \%$ paraformaldehyde overnight at $4{ }^{\circ} \mathrm{C}$. Brains were embedded in $5 \%$ low-melting point agarose (Sigma, St. Louis, MO) and sectioned the following day at $100 \mu \mathrm{m}$ using a vibrating microtome (Technical Products International, St. Louis, MO). Sections were washed in phosphate-buffered saline with $0.5 \%$ Triton X-100 (PBST), then blocked for 1 hour (2\% IgG free bovine serum albumen (BSA), Jackson ImmunoResearch, West Grove, PA) and incubated overnight at room temperature with primary antibody (1:4000 rabbit anti-5HT, Immunostar, Hudson, WI; 1:500 rabbit anti-GABA Sigma, St. Louis, MO; 1:500 rabbit antihistamine, Immunostar, Hudson, WI) in PBST with $0.5 \%$ sodium azide (PBSAT) and 1\% Triton X-100. The following day, sections were washed, blocked and then incubated overnight at room temperature with Cy3-labeled secondary antibody (1:500 goat anti-rabbit; Jackson Immunoresearch, West Grove, PA) in PBST with 0.5\% TX-100. The next day, sections were washed in PBS, cleared in an ascending glycerol series $(40 \%, 60 \%, 80 \%)$ and mounted on glass slides in $80 \%$ glycerol to be viewed and recorded using a Zeiss Pascal confocal attached to a Zeiss Axioplan microscope.

The 5HT antiserum from Immunostar exhibited no cross-reactivity to 5-hydroxytryptophan, 5-hydroxyindole, 5-hydroxyindole-3-acetic acid, or dopamine in Bn-SA/HRP labeling assays (Immunostar Inc. histochemical serotonin antisera specification sheet). Preadsorption of the 5HT-antiserum against BSA (against which this antibody was raised) did not affect labeling (Dacks et al., 2006). To test the specificity of the 5HT antiserum in bumblebee tissue, 5HT antiserum was pre-adsorbed with a 5HT-BSA conjugate (Immunostar) at a concentration of $40 \mathrm{mg} / \mathrm{mL}$ of antibody at a final dilution of 1:4,000, $1: 10,000,1: 20,000,1: 40,000$ and 1:80,000. The 5HT-BSA pre-adsorption left only very weak labeling at antibody dilutions of 1:4,000 and 1:10,000, while at dilutions of 1:20,000, 1:40,000 and 1:80,000 all labeling by the 5HT antiserum was abolished (Supplemental fig. $1)$.

The histamine antiserum from Immunostar exhibited no cross-reactivity to L-histidine or Lhistidine containing peptides and all staining with this antiserum was blocked with preadsorption the histamine conjugate (Immunostar Inc. histochemical histamine antisera specification sheet). In addition, the histamine immunolabeling in Bombus impatiens is very similar to labeling found in many other arthropod species, including Drosophila melanogaster (Bornhauser and Meyer, 1997; Melzig et al., 1996) in which histamine labeling is eliminated in null lines for the gene coding for histidine carboxylase (Melzig et al., 1996).

The GABA antiserum was affinity purified and characterized by a dot-blot immunoassay by Sigma and showed positive binding to GABA and GABA/BSA, but not to BSA alone (Sigma GABA antisera product information sheet). Because all of the transmitters examined 
in this study are small amines that are invariant in structure across all animal species, specificity assays performed on the antibodies used should bear relevance for bumblebees.

\section{Intracellular recording procedure}

Bees were cold anesthetized and immobilized in plastic tubes and the brain was exposed for recording, with insect saline (Baumann and Hadjilazaro, 1972) applied at intervals. Borosilicate thin-walled glass electrodes (tip resistances: 70-120 M $\Omega$ with $1 \mathrm{M} \mathrm{KCl}$ ) were pulled from capillary tubes (A-M Systems, Inc., Carlsborg, WA) on a laser based micropipette puller (model P-2000, Sutter Instruments, Inc., Novato, CA). Micropipettes were backfilled with either: Lucifer yellow $(200 \mu \mathrm{M}$ in $0.5 \mathrm{M} \mathrm{LiCl})$ or one of three Alexa Fluor $^{\circledR}$ hydrazide dyes (excitation spectra at 568,633 , or $647 \mathrm{~nm}, 200 \mu \mathrm{M}$ in $1 \mathrm{M} \mathrm{KCl}$, Molecular Probes, Invitrogen). The intracellular signals of spiking neurons were amplified (Neuroprobe 1600; A-M Systems, Inc.), and recorded on a computer using a Power Lab data acquisition board (ADInstruments, Inc., Colorado Springs, CO). Data were captured at 20 $\mathrm{kHz}$ and saved for offline analysis. The bee was presented with an array of visual stimuli (see below). At the end of the experiment, the neuron was labeled with the dye by injecting a current of -2 to $-5 \mathrm{nA}$ at $1 \mathrm{~Hz}$ for $1-3$ minutes. The brain tissue was then processed as described above for fluorescent labeled brains.

\section{Visual Stimuli}

Color and motion stimuli as described in Paulk and Gronenberg (2008) and Paulk et al. (2008) were presented to the bees' right eye, ipsilateral to the side of the recording. The stimuli are briefly described below.

Color stimuli-An array of 60-light emitting diodes (LED; 20 each violet (v), blue (b), and green (g); peak wavelengths: v: $404 \mathrm{~nm} ; \mathrm{b}: 476 \mathrm{~nm}$; g: $561 \mathrm{~nm}$, with an average halfpeak width: $22.13 \pm 8.86 \mathrm{~nm}$ ) provided colored light flashes. The LEDs' efficiency with respect to the bumblebee spectral sensitivity as well as the behavioral relevance of the applied stimulus intensities is discussed in detail in Paulk and Gronenberg (2008). These LEDs allowed stimulation and discrimination of all three spectral photoreceptor types of bumblebees, although not necessarily at their respective peak sensitivities. The intensities of all three colors were equal (450 photons/count per LED) and the illuminance at the bee's eye (about 1400 lux) corresponded to a level that a human observer would experience under overcast skies.

The LED array was arranged in a half-circle such that the long axis of the array aligned with the long axis of the bee eye, spanning $2.9^{\circ}$ by $123.3^{\circ}$ of the visual field (Paulk et al., 2008). Chromatic sensitivity of neurons was tested using individual arrays of $\mathrm{v}, \mathrm{b}$, or g LEDs or four different combinations (bg, bv, gv, and bgv). Each stimulus was presented as five consecutive 500-ms LED light flashes at $1 \mathrm{~Hz}$.

Motion stimuli-Motion stimuli were presented on a computer-controlled (Vision Egg software; Straw et al., 2006) cathode ray tube (CRT) monitor which subtended $114.0^{\circ}$ by $103.1^{\circ}$ of the visual field of one eye (vertical refresh rate of $160 \mathrm{~Hz}$; Gateway 2000, EV900). At the illuminance levels used (ca. 330 lux), the bee's photoreceptors were not affected by 
the monitor's fast refresh rate (for details of the motion stimuli, see Paulk and Gronenberg, 2008 and Paulk et al., 2008).

Four types of motion stimuli were tested: a wide field high contrast square wave moving grating (filling the entire computer screen, at a spatial frequency of $12.89^{\circ}$, moving at a rate of $57.2^{\circ} / \mathrm{sec}$ ), a small moving bar (subtending $3.85^{\circ}$ by $16.2^{\circ}$, moving at a rate of $73.57^{\circ} \%$ sec), a large moving bar (either a black bar on white or a white bar on black; subtending $9.04^{\circ}$ by $103.1^{\circ}$, moving at a rate of $54.25^{\circ} / \mathrm{sec}$ ), and a moving black edge on a white background (filling the entire computer screen, moving at a rate of $87.69^{\circ} / \mathrm{sec}$ ). Each stimulus was moved in eight different directions (up, down, left, right and $45^{\circ}$ to these respective directions) and four different orientations (Paulk and Gronenberg, 2008; Paulk et al., 2008). Luminance changes of the CRT monitor resulting from the visual stimuli were measured by a photodiode and recorded with the neuronal signals.

\section{Analysis of Responses to Visual Stimuli}

Membrane potential data were imported into Matlab, down-sampled to a resolution of 0.1 $\mathrm{ms}$, and filtered to remove high frequency $(>2000 \mathrm{~Hz})$ and low frequency $(<50 \mathrm{~Hz})$ noise. Electrical noise from the LED relay switches was also removed digitally. The time and mean frequency of the action potentials were measured by detecting spikes above a set threshold. These spike times were used to calculate the coefficient of variation $(\mathrm{CV}=$ standard deviation of the inter-spike intervals/mean value of the inter-spike intervals) during background activity.

A significant response to a visual cue was defined as a change in spike rate greater than 2 times above or below the standard deviation of the mean background spiking activity. Definitions of response categories are provided in the Results. Responses to individual presentations of a stimulus were used to determine each neurons' chromatic and motion preferences, and responses to trains of light flashes were used to analyze the temporal characteristics of the response of each cell. The temporal characteristics that we analyzed were: temporal dynamics of the response and adaptation. Adaptation was identified when a neuron changed its response to the first light flash compared to the subsequent light flashes.

\section{RESULTS}

In the brain of the bumblebee (Bombus impatiens), we intracellularly recorded and morphologically reconstructed 27 neurons with arborizations in the medulla. Here, we first describe the overall anatomy of the bumblebee medulla based on our neuroanatomical and immunocytochemical examinations. We then describe the branching patterns of individually characterized neurons and how they compare with the layers in the medulla. Finally, we describe the physiological properties of the recorded neurons, particularly in terms of how they respond to chromatic cues, and examine how those properties reflect the morphology of the individual neurons with respect to the location of their branching within the medulla.

\section{The medulla is subdivided into eight layers}

We performed Bodian reduced silver staining (Fig. 1A, B) to confirm the high degree of similarity of the bumblebee medulla with that of honeybees as described by Ribi and Scheel 
(1981). The medulla is composed of a mixture of columnar fiber bundles (radial processes) that are crossed by a series of horizontal neurites (tangential elements) at different depths in the medulla, subdividing the medulla into eight layers, as observed in the honeybee (Fig. 1B; Ribi and Scheel, 1981). Medulla layers 1a, 1b, and 2 receive input from lamina monopolar cells and axons of the short-wavelength photoreceptors (R7-R9) in the retina (Fig. 1; Ribi, 1975a, 1975b; Ribi and Scheel, 1981).

Using these landmark strata, we examined the distribution of GABA-like, serotonin-like, and histamine-like immunoreactivity (hereafter called GABA-ir, 5HT-ir, and HA-ir) in the bumblebee medulla to find out whether the different layers exhibited differences in neurotransmitter distribution (Fig. 1C, D, E) suggesting potential functional differences between medulla layers. We found 5HT-ir almost exclusively concentrated in the medulla layers 4 and 5a (Fig. 1C). However, the GABA-ir was observed in all medulla layers, with increased relative levels in layers 1a, 2, in subdivisions of layers 4, 5a, and 6 (Fig. 1D). The retina and, in particular, the lamina exhibit high levels of histamine (Fig. 1E), presumably due to histaminergic photoreceptor input (Bornhauser and Meyer, 1997; Hardie, 1987). We also found a number of HA-ir processes projecting through the outer chiasm into layers 1-3 in the medulla (Fig. 1E), which likely originate from photoreceptors R7-R9, a subset of photoreceptors which bypass the lamina and branch in the medulla (Fig. 1E, G; Fischbach and Dittrich, 1989; Morante and Desplan, 2008; Ribi, 1975a, 1975b; Ribi and Scheel, 1981). In addition to the inputs from the retina, horizontal processes in medulla layer 3 also demonstrated HA-ir (Fig. 1E).

To further examine the photoreceptor inputs, we performed mass fills of photoreceptor neurons from the retina (Fig. 1G). Closer examination of the photoreceptor terminals revealed endings that were similar to the R7-R8 terminals of the honey bee (Fig. 1F, G; Ribi, 1975a). This is illustrated in a schematic representation of the neural reconstructions of the photoreceptors (purple), the lamina monopolar cells (green), and a transmedullary neuron (black) within the context of the eight medulla layers, adapted from Ribi (1975a; Fig. 1F). We found that the photoreceptor and the lamina monopolar neurons (not shown) ended at or distal to layer 1-3, as reported by Ribi (1975a), which contrasted from other medullar elements, including neurons spanning the inner and outer layers or others branching horizontally within layers (Fig. 1H).

To observe anatomical differences among groups of individual neurons, we retrogradely mass-filled medulla neurons from cell bodies situated between the mushroom body calyx and the medulla (asterisk in Fig. 1Hi). Besides tangential elements concentrated in layers 4, 5 , and 6, we found columnar projections reaching through all the layers which were distinguishable on the level of individual neurons (Fig. 1Hii).

\section{Individual medulla neurons represent three major classes}

While the bee medulla contains more than 20 neural classes (Ehmer and Gronenberg, 2002; Ribi and Scheel, 1981), we recorded neurons which could be classified into three broad morphological categories based on their branching patterns in the medulla and their projections outside of the medulla $(n=27)$. The first category included 15 neurons that had wide branches across large portions of the medulla and which project out of the medulla to 
either the protocerebrum or to the contralateral medulla, a group we refer to as large field medulla neurons (Fig. 2). The second category included seven neurons that had widelybranching neurites that remained within the medulla, which we refer to as amacrine medulla neurons (Fig. 3). Finally, the third category of neurons included five columnar medulla neurons, which we referred to as transmedullary neurons (Fig. 3).

The number of neurons recorded in each category likely does not reflect the abundance of neurons in each category as the diversity in cell size likely affected our ability to obtain intracellular recordings. Instead, the neurons we sampled likely related more directly to the relative sizes of the neurons. The relatively smaller, but significantly more abundant, columnar neurons were particularly difficult to impale compared to the horizontal or tangential elements, such as the amacrine or large field medulla neurons. While these categories exhibited gross morphological similarities, within each category the neurons exhibited diverse morphological and physiological characteristics, indicating that many of the individual neurons could be representative of distinct morphological classes. This is primarily a result of the intracellular recording technique, which resulted in sampling neurons almost at random. However, a unifying aspect of these classes is that each set of neurons occupied specific layers within the medulla.

Large field medulla neurons-All of the large field medulla neurons showed arrays of fine collaterals covering considerable areas of the medulla (ranging from 100-300 $\mu \mathrm{m}$ widths) and exited the medulla via the posterior optic commissure (POC) and projected posteriorly into the protocerebrum (Fig 2; Hertel et al., 1987). The majority of large field medulla neurons branched in layers 4-6 (Fig. 2F-I, 3F), with only two of the fifteen neurons producing arborizations in layers 1-3 (Fig. 2E,F, 3F; Table 2). The large field neurons that were completely filled $(n=9)$, either projected diffusely into the posterior protocerebrum $(n=4$; Fig. $2 A)$, into both the contralateral medulla and the posterior protocerebrum $(n=4$; not shown), or into the mushroom body calyces ( $\mathrm{n}=1$; not shown; see: Paulk and Gronenberg, 2008; Table 2). For all the large field medulla neurons found except one (Fig. 2B,E), the side of the recording was the same side we found fine branching patterns in the medulla, which were possibly the input, or postsynaptic, regions of the neurons (Fig. 2A,C,G,H). The single exception to this was a neuron which produced varicosities all around the medulla, possibly indicating that the neuron was sending outputs into the side of the recording (Fig. 2B,E). The cell bodies (when filled; $n=8$ ) were either situated lateral to the lateral mushroom body calyx on the same side of the recording ( $n=5$; not shown) or were situated medially, above the central complex ( $n=3$; Fig. 2A; Table 2).

Amacrine neurons-Amacrine medulla neurons $(\mathrm{n}=7)$ by definition have neurites confined to the medulla (Fig. 3C). Many of these local interneurons were confined to layers 4 and 5 (n=4; Fig. 2H, I, 3F) and three of the neurons extended into other layers (Table 2). The amacrine neurons had branching patterns which extended horizontally within the layers, often spanning an oval-shaped field within the medulla around 100-200 $\mu \mathrm{m}$ in diameter. When their cell bodies were filled $(n=5)$, the cell bodies were lateral to the mushroom body calyx, next to the optic lobes (Table 2). 
Transmedullary neurons-The transmedullary neurons recorded in this study ( $\mathrm{n}=5$ ) resembled the columnar neurons shown in Fig. 1G, with fine dendritic branching in the medulla and projected via the inner optic chiasm to the lobula (Fig. 3A, B, D). Unlike most of the amacrine and large field medulla neurons, the recorded transmedullary neurons sent fine processes into layer 1, sometimes 2, and rarely into layers 3-6 (Fig. 3A, B, D, F; Table 1). The transmedullary neural cell bodies, when filled, were located distal to the medulla, near the outer optic chiasm (Fig. 3A, D). All of the transmedullary neurons were similar to previously defined transmedullary categories 1, 4, or 7 in bees (Ribi and Scheel, 1981) or transmedullary categories 1, 1a, or 9 in flies (Fischbach and Dittrich, 1989). These similarities to three previously identified neural classes (out of nearly 10 types in the bee and 15 in the fly; Ribi and Scheel, 1981; Fischbach and Dittrich, 1989) indicated that the recorded transmedullary neurons appeared to belong to one or a few categories of neurons out of the many defined transmedullary types based on morphology.

\section{Physiological response properties}

The basic physiological properties of the medulla neurons did not differ significantly among the three categories. Spontaneous firing rates for each neural class were not significantly different (Mann-Whitney $U$-test, $\mathrm{p}>0.05$ for all comparisons) and there were no significant differences in coefficient of variation values between the groups (Mann-Whitney $U$-test, p $>0.05$ for all comparisons) confirming there were no differences in the temporal aspects of the spontaneous activity between the neural classes. Although the primary focus of this study was on the color responses of these neurons, we cursorily tested motion stimuli for a subset of the neurons examined. Of the neurons tested for motion cues $(n=7)$, three medulla neurons did not respond to motion cues at all, three neurons were non-directionally motion sensitive and one neuron was directionally motion sensitive (not shown; Table 3). Among those neurons we were able to test for receptive fields $(n=7)$, five neurons had wide receptive fields, one had a small receptive field, and one neuron had a complex receptive field, showing different responses to stimuli in different parts of the eye (Fig. 5F; Table 3). When measured, receptive field size correlated with the assumed dendritic field size: the (anatomically) large field neurons had large receptive fields while one neuron with a small receptive field was an amacrine neuron.

\section{Responses to light flashes differ among medulla neuron categories and among layers}

We observed at least four very different response types to a series of five chromatic light flashes exhibited by the medulla neurons recorded in this study (Fig. 4). These response types included phasic-tonic excitatory responses, in which there was a burst of activity at the onset of the light flash followed by decreased activity for the duration of the light flash (Fig. $4 \mathrm{~A})$. Other neurons produced phasic responses to the onset of the light flash exclusively (Fig. 4B). On-off responses were identified when the neurons produced a burst of activity at the onset and the offset of the light flashes (Fig. 4C). Finally, we found medulla neurons that were tonically inhibited followed by a rebound excitation (Fig. 4D). Interestingly, the neuron depicted in figure 4D transiently increased its membrane potential at the beginning of the light-induced inhibition, suggesting a combination of phasic excitatory and tonic inhibitory input. 
The three anatomical classes of medulla neurons differed in the temporal response types they were most likely to exhibit (Fig. 7A). For example, 4 of 5 transmedullary neurons exhibited an inhibitory response followed by rebound excitation (inhibition-off; Fig. 7A), while 8 out of 15 of the large field medulla neurons had phasic-tonic responses (Fig. 7A). The amacrine medulla neurons showed the least preference for particular response types (Fig. 7A). Hence, the recorded medulla neurons demonstrated a range of temporal response properties to light flashes that differed across the three anatomical types of neurons.

In addition to the three major neuron categories, we compared response properties across neurons with arborizations in different medulla layers. When comparing the neurons with arborizations in medulla layers $1-3$ to those with arborizations in layers 4-5, we found remarkable differences among the recorded neurons (Fig. 7B): the phasic-tonic response type was observed more often in the neurons branching in layers 4-5 ( $\mathrm{n}=9$ of 17 tested) while the tonic inhibition with rebound excitation response type dominated in neurons with branching in layers $1-3$ ( $n=6$ of 10 tested). Therefore, the association of (presumed input) arborizations with a particular medulla layer seemed to significantly contribute to a neuron's response properties.

\section{Medulla neurons exhibit a wide variety of chromatic responses}

The neurons exhibited a range of chromatic sensitivities, which generally fell into three categories, as defined by previous investigators (Kien and Menzel, 1977a, 1977b). They included broad-band (or achromatic) responses, where the neurons responded to the three LED colors in the same way (Fig. 5A); narrow-band responses, where the neurons responded to only one or two LED colors (Fig. 5B, C); or color opponent responses (Fig. $5 \mathrm{D})$ where neurons responded antagonistically to different wavelengths or where the presence of one color changed the response of the neuron to another color (Fig. 5D).

As mentioned in the Methods, we could deduce what photoreceptor types input to the individual medulla neurons based on their responses to the LEDs. This information is included in Table 3. For example, narrow-band neurons may have been activated by all three LED colors, but had stronger responses to one LED type over the other, such as having a stronger response to violet (Fig. 5B) or blue LEDs (Fig. 5C) compared to the other LED colors. In these cases, the neurons were likely only receiving major input in one color channel (such as ultraviolet (Fig. 5B) or blue photoreceptor types (Fig. 5C); Table 3), but since the LED colors overlapped somewhat with the other photoreceptor types, we observed some small responses to the other LED colors (Fig. 5B, C). Comparing the neurons' responses to different colors showed that most of the medulla neurons received excitatory inputs from the three photoreceptor types, while some received inhibitory inputs (Table 3).

When we examined color opponent neurons, we found that 4 of the 5 color opponent medulla neurons received either excitatory or inhibitory input from all three color channels, namely ultraviolet, green, and blue photoreceptor types (Fig. 5D; Table 3). The essential aspect of differentiating their responses was to examine how the different LED colors affected the neurons' response dynamics. For example, the medulla neuron in Fig. 5D, F showed a tonic response to violet, a phasic response to blue, and no clear response to green. The tonic response to violet disappeared with the simultaneous presentation of blue, when 
the cell only produced a phasic response (gray arrow; Fig. 5D). Surprisingly, the tonic response was also replaced with a phasic response upon the simultaneous presentation of green and violet (gray arrow; Fig. 5D), which indicated that, while green light alone did not appear to change the neuron's spike rate, it could nevertheless influence the cell's response dynamics. In addition, the inclusion of green or blue in the stimulus reduced the latency of the response compared to during only violet light presentations (dotted line; Fig. 5D).

In some medulla neurons, we found that response dynamics changed with different colors of the light flash (Fig. 5E; Table 2). In the example in Fig. 5E, the medulla neuron demonstrated a phasic response to green with an 'off' response (arrow), a phasic response to blue without an 'off' response, and a phasic-tonic response to violet, also without an off response (arrow). We found that 4 of the 15 tested large field neurons and two of the amacrine neurons changed their response types based on the light flash color.

In addition, we observed a complex receptive field in one amacrine neuron which related to the color of the stimulus (Fig. 5F). The neuron responded to stimuli across most of the eye, but exhibited different temporal dynamics in response to violet light. For instance, it produced a phasic burst at the onset of the light flash when the stimulus was in the middle to upper portion of the visual field (Fig. 5F). However, violet light presented immediately below this area induced a delayed response. A mixture of these two responses was present during presentations of violet light across the eye, indicating some complex interactions between violet stimuli presented to different areas of the eye (Fig. 5F). We did not observe this same pattern when we presented blue or green light (not shown).

When color sensitivity was compared between neural categories, amacrine and large field medulla neurons had similar proportions of broad-band, narrow-band, and color opponent responses, indicating that color sensitivity was broadly distributed among medulla neuron types (Fig. 7A). This result was not surprising considering the diversity of morphologies found among the amacrine and large field medulla neurons. While only three transmedullary neurons were tested with the full range of LED colors, one was found to be narrow band (Fig. 5C), while the remaining two neurons were broad band sensitive. These physiological differences did not appear to correlate with the morphology, as the neurons had similar branching patterns.

Yet, when we considered how the neurons were distributed among the medulla layers, we found remarkable differences between the neurons which primarily branch in layers 1-3 versus those mostly branching in layers 4-5 (Fig. 7B). The neurons branching in layers 1-3 were predominantly broad-band ( 5 of 7 tested) while neurons with branching patterns in layers 4-5 had relatively even distributions of broad-band, narrow-band and color opponent responses (Fig. 7B). Therefore, color sensitivity could be correlated with the location of the neurons in the medulla layers.Based on how the neurons responded to each of the colors, we could deduce how many of each of the neurons received excitatory or inhibitory input from each of the photoreceptor types, which is listed in Table 3. Most of the medulla neurons received excitatory inputs from the three photoreceptor types, while some received inhibitory inputs (Table 3). 


\section{Medulla neurons exhibit different amounts of adaptation}

The three anatomical types of neurons also had differences in adaptation in response to the series of five LED light flashes (Fig. 6). We defined adaptation as a change in the response of the neuron to the first versus subsequent light flashes (Fig. 6C, D). While 15 of the 27 neurons did not change their responses over time (Fig. 6A, B), twelve medulla neurons did adapt to the light flashes (Fig. 6C, D; Table 3). Some neurons reduced their responses or did not respond at all to subsequent light flashes (Fig. 5D to violet, 6C), while other neurons exhibited complex types of adaptation to repeated light flashes (Fig. 6D). For instance, Fig. $6 \mathrm{D}$ depicts the excitatory 'on' response of a large field medulla neuron decrease from the first to subsequent light flashes while the inhibitory 'off' responses increased in magnitude over time (black arrows; Fig. 6D). This change in the inhibitory responses was observed across all seven trials of five sequential light flashes and indicated that some medulla neurons exhibited complex adaptations to stimuli in general. Interestingly, only one of the transmedullary neurons adapted to light flashes while nearly half of the amacrine and the large field medulla neurons did so (Table 3; Fig. 7A, B). In addition, nearly $30 \%$ of the neurons occupying layers $1-3$ adapted while $47 \%$ of the neurons branching in layers 4-5 adapted.

\section{DISCUSSION}

The medulla is a key processing stage in insect vision, where information from the retina and the lamina is processed, sorted, and sent on to specific areas of the insect brain. We have characterized examples of medulla neurons in the bumble bee brain that correspond to three basic morphological types of neurons (Fig. 3E, F, 7). Not surprisingly, our sample of neurons revealed an array of different physiological response types (Fig. 7). The medulla contains the largest number of neurons of all the optic neuropils, and it is characterized by a variety of morphological types (Ribi and Scheel, 1981; Strausfeld, 1976) that significantly contribute to visual information processing. Considering the spatial, temporal and chromatic complexity of natural visual scenes, one might expect a wide diversity of neurons and physiological response properties necessary to enable a bee's many different behaviors (e.g. during flight or foraging). While the anatomical and physiological diversity of this neuropil makes it difficult to construct a global framework of medulla function, some general principles of the functional organization of this brain structure are becoming apparent.

\section{Medulla anatomy}

The bumblebee medulla contains layers that are delineated by the horizontal neurites crossing the numerous columnar medulla neurons (Fig. 1; Ribi and Scheel, 1981; Strausfeld, 1976). These layers were delineated both by the neural branching patterns and by GABA, histamine, and serotonin immunolabeling. In particular, layers 4 and 5a correlated well with serotonin staining and with complex layering of GABAergic fibers (Fig. 1) suggesting that neuronal processes in these layers are subject to spatially restricted inhibition and neuromodulatory control. Most of the tangential neurons we recorded from had their major branches in layers 4 and 5a [Fig. 2E-G; the "serpentine layer" (Ehmer and Gronenberg, 2002; Strausfeld, 1976) that separated the outer from the inner medulla (Strausfeld, 2005)]. Nearly all of the large field medulla neurons had processes in the serpentine layer, which 
means that the computational operations occurring in the serpentine layer could be pivotal to understanding how the medulla directly interfaces with the protocerebrum.

In contrast, much of the histamine labeling was present in the outer medulla and likely correlates to the short wavelength photoreceptor input (Fig. 1E-G). The histaminergic photoreceptor and lamina monopolar cell terminals could provide inhibitory inputs to the neurons branching in the outer medulla (layers 1-3), causing inhibitory-off responses in neurons of the outer medulla (such as transmedullary neurons)(Fig. 4D, 7). Therefore, the histamine labeling combined with the physiological response properties of neurons in the outer medulla could point possible functional connections between the photoreceptors and the transmedullary neurons.

\section{Color sensitive responses}

Our results suggest that a major function of the medulla is color processing. It involves the integration of information originating from different photoreceptor types sensitive to specific wavelengths and the production of responses specific to the wavelength presented. The basic stages of color processing begin with comparing the inputs from the different photoreceptor types. The best candidate region for the initial stages of color processing in the bee brain is the medulla as it receives direct input from blue and ultraviolet photoreceptors as well as the outputs from lamina monopolar cells, which represent the green photoreceptor input processed by the lamina (Ribi, 1975a, 1975b; Ribi and Scheel, 1981). Therefore, it is not surprising that we found that medulla neurons exhibit a wide range of color sensitive responses.

In different medulla neurons, we encountered broad-band (achromatic), narrow-band, and color opponent response types (Fig. 5, 7; Hertel, 1980). By comparing published data with our present data set, we may be able to address a very basic question: where in the medulla are the initial stages of color processing performed? We know that the blue and ultraviolet photoreceptors provide input to layers 1 and 2 alongside green-sensitive laminar inputs to the medulla (Ribi, 1975a). Many of the transmedullary neurons had projections in layers 1 and 2, suggesting that the neurons with branches in the distal medulla layers receive direct input from the photoreceptors and the lamina monopolar cells. This input from the primary color channels to layers 1 and 2 is essential for both broad-band (integrating all three color channels) and narrow band responses (resulting from selective interaction between one or two color channels), as reflected by the response properties of the few transmedullary neurons we were able to record from. Hence the retinotopic (small-field) output from the medulla provides both, achromatic and color specific information for parallel processing at the next level, the lobula.

The next stage of medullar processing involves producing color opponent responses, which requires a complex integration of both inhibitory and excitatory interactions of the three color channels. The primary candidates for this type of processing are the amacrine and large-field medulla neurons. Color opponency and narrow- and broad-band color sensitivity have been found for large field and amacrine (intrinsic) medulla neuron types in honeybees (Fig. 5, 7; Hertel, 1980; Hertel and Maronde, 1987; Hertel et al., 1987). The mechanism behind color sensitivity could involve the inputs of the transmedullary neurons which project 
from layers 1 and 2 to layers 4 and $5 \mathrm{a}$ and the large field and amacrine neurons. These transmedullary inputs would need to have different balances of excitatory and inhibitory inputs to the amacrine and large field neurons to be able to produce color opponent responses. Thus, neurons in layers 1 and 2 may be involved in signaling color along a narrow- or broad-band pathway and may then input to neurons in the serpentine layer (layers 4-5a), which integrate the signals to produce color opponent responses. In addition, it should be noted that the amacrine neurons are more likely the effectors of color opponency since they can compare the paths of information across columnar elements, which is why it is not surprising to find color sensitivity in the amacrine neurons.

Interestingly, of the recorded large field and amacrine neurons, the neurons which branched in layers 1,2 , or 3 were broad-band or narrow-band. We only found color opponency when we recorded from neurons with projections in the serpentine layer, or layers 4 and $5 \mathrm{a}$, as was also observed by Hertel (1980). In addition, the fact that there were relatively high levels of GABAergic and serotonergic staining in layers 4 and 5a hints at the possibility that GABA and serotonin could play a role in the color opponent and temporal coding of color exhibited by the medulla neurons. For instance, serotonin could be involved in modulating the temporal responses to color or decrease the activity of specific types of neurons mediating the modulation of visual behaviors that results from serotonin treatment (Erber and Kloppenburg, 1995; Kloppenburg and Erber, 1995). On the other hand, GABA could be involved in producing the color opponent responses, which rely on inhibitory interactions between colors (Fig. 5D).

Therefore, we propose that the layered organization of the medulla represents multiple stages of color processing in a single structure, with the basic levels of color signaling (narrow-band) occurring in the distal layers. As one progresses proximally, the color signaling becomes more complex, enabling color opponency. This system has been proposed in the fly visual system based on anatomical findings (Douglass and Strausfeld, 1998). Our study is the first account addressing color responses that supports this concept by physiological data.

\section{Temporal aspects of the medulla neuron responses}

Many of the medulla neurons we recorded exhibited a range of temporal response types to light flashes. For individual neurons the response type elicited often depended on the color of the stimulus (Fig. 5E), which has been observed previously in honeybees (Hertel, 1980; Hertel and Maronde, 1987; Hertel et al., 1987). This aspect of the medulla neurons' color sensitivity could be essential to understanding visual processing in the bee brain. By producing different temporal response types based on color, a neuron would produce an 'onoff' response for some colors and a simple 'on' response for others. Instead of simply encoding color in spike rate, some medulla neurons appear to encode color in the timing of individual spikes, which adds an additional layer of complexity to the encoding of color. This probably also allows processing of color information using a smaller number of neurons as each individual neuron can represent different colors or color combinations, depending on the timing of the response compared to other neurons. This additional 
temporal coding ability might be particularly advantageous for a small insect brain where neuron numbers are limited.

\section{Parallel streams of information: comparison with lobula neurons}

While the major visual pathway proceeds from the medulla to the lobula, the majority of the neurons we recorded in the medulla projected to the protocerebrum via the posterior optic commissure (POC; Fig. 7), bypassing the lobula. We know that lobula neurons also send output to the protocerebrum (DeVoe et al., 1982; Hertel, 1980; Hertel and Maronde, 1987; Ribi and Scheel, 1981). Therefore, we have two parallel streams of information exiting the optic lobes and projecting to the protocerebrum: one directly originating in the medulla, bypassing the lobula, and the other leaving the lobula. A third visual pathway includes the ocelli, secondary visual structures located at the dorsal surface of the bee head, which have been shown to feed into the posterior protocerebrum. Ocellar inputs have been shown to exhibit light and dark responses and, only recently, color-based directional sensitivity (Milde, 1981; Pan and Goodman, 1977; van Kleef et al., 2008). In addition, there are parallel streams of information from the lobula and the medulla to the mushroom bodies (paired structures associated with learning and memory; Heisenberg, 1998, 2003). These streams of information from the medulla and the lobula are segregated within the mushroom bodies (Ehmer and Gronenberg, 2002; Gronenberg, 1986; Paulk and Gronenberg, 2008).

In terms of target areas in the central brain, the medulla protocerebral output appear to overlap with posterior lobula output, which begs the question; why are there parallel streams of information from the medulla, lobula, and the ocelli to similar areas in the central brain? The answer likely relates to the type of information sent to these brain regions. The lobula outputs to the posterior protocerebrum are largely motion sensitive and are broad-band color sensitive (hence color blind; Paulk et al., 2008). The medulla outputs, however, are often color selective and not necessarily motion sensitive (Fig. 7; Hertel, 1980). In addition, the medulla neurons may be participating in sampling the visual scene for edges and contrast, particularly if they are spatially antagonistic (Fig. 5F; Hertel, 1980; Hertel et al., 1987). Therefore, in the same regions of the posterior protocerebrum, there may be information converging from motion and color pathways. This convergence could be essential for behavior as the posterior protocerebrum contains descending neurons that send input to premotor centers in the bee nervous system (Ibbotson, 2001). Therefore, the convergence of the functionally different lobula and medulla neurons on the posterior protocerebrum could be essential for the bee to perform its normal behaviors.

\section{Supplementary Material}

Refer to Web version on PubMed Central for supplementary material.

\section{ACKNOWLEDGEMENTS}

We would like to thank Richard Levine, Jean-Marc Fellous, James Phillips-Portillo, Andre Riveros, Charles Higgins, and Thomas Christensen for their helpful comments on the manuscript. We would also like to thank Jennifer Vega, Tulika Balagopal and Jorge Palacios for providing dye-fills and technical support and James PhillipsPortillo for providing the Bodian preparations. 
The project described was supported by Grant Number F31NS051123 from the National Institute of Neurological Disorders And Stroke to A.C.P. and NSF Grant IOB-0519483 to W.G. The content is solely the responsibility of the authors and does not necessarily represent the official views of the National Institute of Neurological Disorders and Stroke or the National Institutes of Health or the National Science Foundation. Research was additionally supported by NIH Grant Number T32 AG007434, and by the Center for Insect Science, University of Arizona.

\section{LITERATURE CITED}

Baumann F, and Hadjilazaro B. 1972 A depolarizing aftereffect of intense light in the drone visual system. Vis Res 12: 17-31. [PubMed: 5034631]

Bornhauser BC, Meyer EP. 1997 Histamine-like immunoreactivity in the visual system and brain of an orthopteran and a hymenopteran insect. Cell Tissue Res 287: 211-221. [PubMed: 9011397]

Callaway EM. 2005 Structure and function of parallel processing pathways in the primate early visual system. J Physiol 566:13-19. [PubMed: 15905213]

Campos-Ortega JA, Strausfeld NJ. 1972 The columnar organization of the second synaptic region of the visual system of Musca domestica L. I. Receptor terminals in the medulla. Z Zellforsch 124:561-582. [PubMed: 5010818]

Dacks AM, Christensen TA, Hildebrand JG. 2006 Phylogeny of a serotonin-immunoreactive neuron in the primary olfactory center of the insect brain. J Comp Neurol 498:727-746. [PubMed: 16927264]

DeVoe RD, Kaiser W, Ohm J, Stone LS. 1982 Horizontal movement detectors of honeybees: directionally-selective visual neurons in the lobula and brain. J Comp Physiol 147:155-170.

De Souza J, Hertel H, Ventura DF, Menzel R. 1992 Response properties of stained monopolar cells in honeybee lamina. J Comp Physiol A 170: 267-274.

Douglass JK, Strausfeld NJ. 1996 Visual motion-detection circuits in flies: parallel direction- and nondirection-sensitive pathways between the medulla and lobula plate. J Neurosci 16: 4551-4562. [PubMed: 8764644]

Douglass JK, Strausfeld NJ. 1998 Functionally and anatomically segregated visual pathways in the lobula complex of a calliphorid fly. J Comp Neurol 396:84-104. [PubMed: 9623889]

Douglass JK, Strausfeld NJ. 2003 Retinotopic pathways providing motion-selective information to the lobula from peripheral elementary motion-detecting circuits. J Comp Neurol 457:326-344. [PubMed: 12561074]

Douglass JK, Strausfeld NJ. 2005 Sign-conserving amacrine neurons in the fly's external plexiform layer. Vis Neurosci 22: 345-358. [PubMed: 16079009]

Douglass JK, Strausfeld NJ. 2007 Diverse speed response properties of motion sensitive neurons in the fly's optic lobe. J Comp Physiol A 193:233-247.

Ehmer B, Gronenberg W. 2002 Segregation of visual input to the mushroom bodies in the honeybee (Apis mellifera). J Comp Neurol 451:362-373. [PubMed: 12210130]

Erber J, Kloppenburg P. 1995 The modulatory effects of serotonin and octopamine in the visual system of the honey bee (Apis mellifera L.) I. Behavioral analysis of the motion-sensitive antennal reflex. J Comp Physiol A 176:111-118.

Fischbach KF, Dittrich APM. 1989 The optic lobe of Drosophila melanogaster. I. A Golgi analysis of wild-type structure. Cell Tissue Res 258: 441-475.

Gilbert C, Penisten DK, DeVoe RD. 1991 Discrimination of visual motion from flicker by identified neurons in the medulla of the fleshfly Sarcophaga bullata. J Comp Physiol A 168: 653-673. [PubMed: 1920163]

Giurfa M 2007 Behavioral and neural analysis of associative learning in the honeybee: a taste from the magic well. J Comp Physiol A 193:801-24.

Gregory CE. 1980 The Bodian Protargol Technique. In: Neuroanatomical Techniques (Strausfeld NJ and Miller TA, ed.) pp. 75-95. Springer-Verlag: New York.

Gribakin FG. 1969 Cellular basis of colour vision in the honey bee. Nature (Lond.) 223: 639-641.

Gribakin FG. 1972 The distribution of the long wave photoreceptors in the compound eye of the honey bee as revealed by selective osmic staining. Vision Res 12: 1225-1230. [PubMed: 4114835]

Gronenberg W 1986 Physiological and anatomical properties of optical input-fibres to the mushroom bodies in the bee brain. J Insect Physiol 32:695-704. 
Hardie RC (1987) Is histamine a neurotransmitter in insect photoreceptors? J Comp Physiol 161: 301213.

Heisenberg M 1998 What do the mushroom bodies do for the insect brain? Learn Mem 5:1-10. [PubMed: 10454369]

Heisenberg M 2003 Mushroom body memoir: from maps to models. Nat Rev Neurosci 4:266-275. [PubMed: 12671643]

Hertel H 1980 Chromatic properties of identified interneurons in the optic lobes of the bee. J Comp Physiol A 137:215-231.

Hertel H, Maronde U. 1987 The physiology and morphology of centrally projecting visual interneurons in the honeybee brain. J Exp Biol 133:301-315.

Hertel H, Schäfer S, Maronde U. 1987 The physiology and morphology of visual commissures in the honeybee brain. J Exp Biol 133:283-300.

Homberg U, Hildebrand JG. 1991 Histamine-immunoreactive neurons in the midbrain and suboesophageal ganglion of sphinx moth Manduca sexta. J Comp Neurol 307:647-57. [PubMed: 1869635]

Homberg U, Würden S. 1997 Movement-sensitive, polarization-sensitive, and light-sensitive neurons of the medulla and accessory medulla of the locust, Schistocerca gregaria 386: 329-346.

Hubel DH, Livingstone MS. 1987 Segregation of form, color, and stereopsis in primate area 18. J Neurosci 7:3378-3415. [PubMed: 2824714]

Ibbotson MR. 2001 Evidence for velocity-tuned motion-sensitive descending neurons in the honeybee. Proc Biol Sci 268:2195-201. [PubMed: 11674866]

Kien J, Menzel R. 1977a Chromatic properties of interneurons in the optic lobes of the bee-I. Broadband neurons. J Comp Physiol 113:17-34.

Kien J, Menzel R. 1977b Chromatic properties of interneurons in the optic lobes of the bee-II. Narrow band and color opponent neurons. J Comp Physiol 113:35-53.

Kloppenburg P, Erber J. 1995 The modulatory effects of serotonin and octopamine in the visual system of the honey bee (Apis mellifera L.) II. Electrophysiological analysis of motion-sensitive neurons in the lobula. J Comp Physiol A 176:119-129.

Melzig J, Buchner S, Wiebel F, Wolf R, Burg M, Pak WL, Buchner E. 1996 Genetic depletion of histamine from the nervous system of Drosophila eliminates specific visual and mechanosensory behavior. J Comp Physiol A 179: 763-773. [PubMed: 8956497]

Menzel R 1974 Spectral Sensitivity of Monopolar Cells in the Bee Lamina. J Comp Physiol 93: 337346.

Menzel R, Giurfa M. 2006 Dimensions of cognition in an insect, the honeybee. Behav Cogn Neurosci Rev 5:24-40.

Milde J (1981) Graded potentials and action potentials in the large ocellar interneurons of the bee. J Comp Physiol 143: 427-434.

Morante J, Desplan C. 2008 The color-vision circuit in the medulla of Drosophila. Curr Biol 18: 553565 [PubMed: 18403201]

Ney-Nifle M, Keasar T, Shmida A. 2001 Location and color learning in bumblebees in a two-phase conditioning experiment. J Insect Behav 14:697-711.

O'Caroll DC, Osorio D, James AC, Bush T. 1992 Local feedback mediated via amacrine cells in the insect optic lobe. J Comp Physiol A 171: 447-455.

Osorio D 1986 Directionally selective cells in the locust medulla. J Comp Physiol A 159: 841-847. [PubMed: 3806440]

Pan KC, Goodman LJ. 1977 Ocellar projections within the central nervous system of the worker honeybee (Apis mellifera). Cell Tissue Res 176: 505-527. [PubMed: 832310]

Paulk AC, Gronenberg W. 2008 Higher order visual input to the mushroom bodies in the bee, Bombus impatiens. Arthropod Struct \& Dev 37: 443-458.

Paulk AC, Phillips-Portillo J, Dacks AM, Fellous JM, Gronenberg W. 2008 The processing of color, motion, and stimulus timing are anatomically segregated in the bumblebee brain. J Neurosci 28:6319-6332. [PubMed: 18562602] 
Peitsch D, Feitz A, Hertel H, de Souza J, Ventura DF, Menzel R. 1992 The spectral input systems of hymenopteran insects and their receptor-based colour vision. J Comp Physiol A 170:23-40. [PubMed: 1573568]

Ribi WA. 1975a The first optic ganglion of the bee. I. Correlation between visual cell types and their terminals in the lamina and medulla. Cell Tiss Res 165:103-111.

Ribi WA. 1975b The neurons in the first optic ganglion of the bee (Apis mellifera) Advan Anat Embryol and Cell Biol 50:1-43. [PubMed: 1199826]

Ribi WA, Scheel M. 1981 The second and third optic ganglia of the worker bee: Golgi studies of the neuronal elements in the medulla and lobula. Cell Tissue Res 221:17-43. [PubMed: 7032703]

Roig-Alsina A, Michener CD. 1993 Studies of the phylogeny and classification of long-tongued bees (Hymenoptera: Apidae). Univ Kansas Sci Bull 55:124-162.

Schultz TR, Engel MS, Ascher JS. 2001 Evidence for the origin of eusociality in the corbiculate bees (Hymenoptera: Apidae). J Kansas Entomol Soc 74:10-16.

Souza JM, Hertel H, Menzel R, Ventura DF. 1987 Marking and recording of monopolar cells in the bee lamina. Braz J Med Biol Res 20: 851-855 [PubMed: 3455267]

Srinivasan MV. 1993 Pattern recognition in the honeybee: recent progress. J Insect Physiol 40:183194.

Srinivasan MV, Zhang SW. 1998 Probing perception in a miniature brain: Pattern recognition and maze navigation in honeybees. Zoology 101:246-259.

Strausfeld NJ. 1976 Atlas of an insect brain Berlin: Springer.

Strausfeld NJ. 1989 Beneath the compound eye: neuroanatomical analysis and physiological correlates in the study of insect vision. In Stavenga DG and Hardie RC (eds.) Facets of vision Heidelberg, New York: Springer pp. 318-359.

Straw AD. 2008 Vision Egg: An Open-Source Library for Realtime Visual Stimulus Generation. Front Neuroinform doi:10.3389/neuro.11.004.2008.

Straw AD, Warrant EJ, O’Carroll DC. 2006 A ‘bright zone' in male hoverfly (Eristalis tenax) eyes and associated faster motion detection and increased contrast sensitivity. J Exp Biol 209: 4339-4354. [PubMed: 17050849]

Van Kleef J, Berry R, Stange G. 2008 Directional selectivity in the simple eye of an insect. J Neurosci 28: 2845-2855. [PubMed: 18337415]

Witthöft W 1967 Absolute Anzahl und Verteilung der Zellen im Hirn der Honigbiene. Z Morph Tiere 61:160-184. 

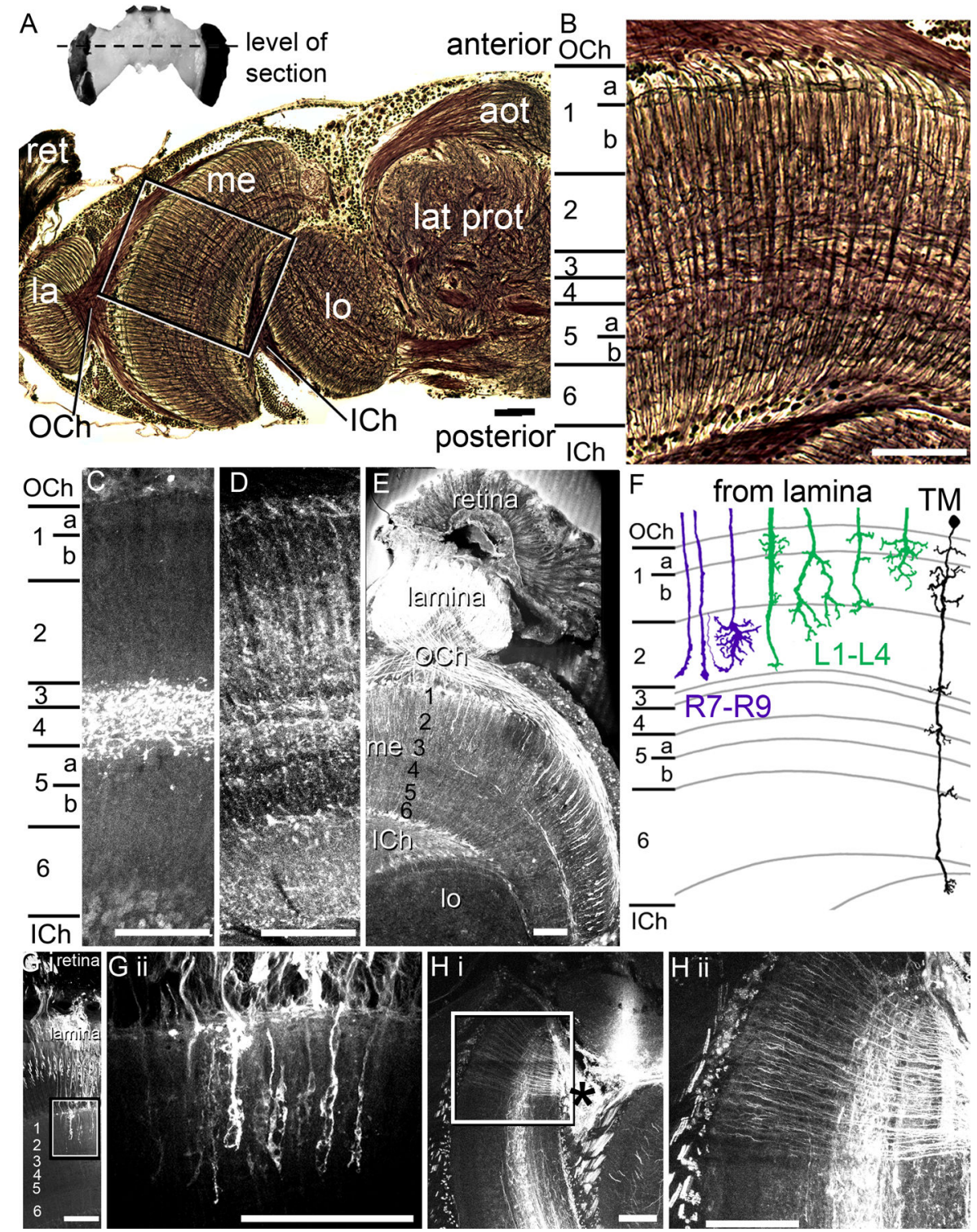

Fig. 1.

Anatomy of the medulla. A. The entire bumblebee brain (above). The dotted line indicates the level of the section taken for the Bodian staining (below). The Bodian section is from half of the bumblebee brain. The retina (ret) inputs to the lamina (la), which then projects via the outer chiasm $(\mathrm{OCh})$ to the medulla (me). Many medulla neurons project via the inner chiasm (ICh) to the lobula (lo). The box indicates the location of where the image was taken for the medulla image in B. Anterior optic tubercle (aot); lateral protocerebrum (lat prot). B. The six layers of the medulla, as defined by Ribi and Scheel (1981). The layers contain a mixture of columnar and horizontal elements arranged in layers. C. Serotonin-like immunolabeling in the medulla indicates that serotonin is concentrated in medulla layer 4 with some labeling in layer 5a. D. GABA-like immunolabeling in the medulla. The GABA distribution in the medulla also follows the layers as defined by Ribi and Scheel (1981), with some layers containing increased amounts of GABA than others (as indicated by the lighter 
bands). E. Histamine-like immunolabeling in the peripheral optic lobes indicates that histamine is localized in the retina, lamina, and in terminals in the outer medulla and in a longitudinal band (layer 3). F. A schematic diagram of the lamina monopolar neurons (green), photoreceptor inputs (purple), and a transmedullary neuron, adapted from Ribi, 1975a. G i. A retrograde tracer applied to the retina in the bee eye filled retina and photoreceptor neurons, which then projected into layers 1-3 in the medulla. The area in the box in $\mathbf{G} \mathbf{i}$ is shown in $\mathbf{G}$ ii. $\mathbf{H}$ i. Retrograde mass fills of medulla neurons from cell bodies near the mushroom body calyces $(*)$ illustrating the mixture of columnar and tangential elements in the medulla. Area boxed in $\mathbf{H} \mathbf{i}$ enlarged in $\mathbf{H}$ ii. Scale bars $=100 \mu \mathrm{m}$. 

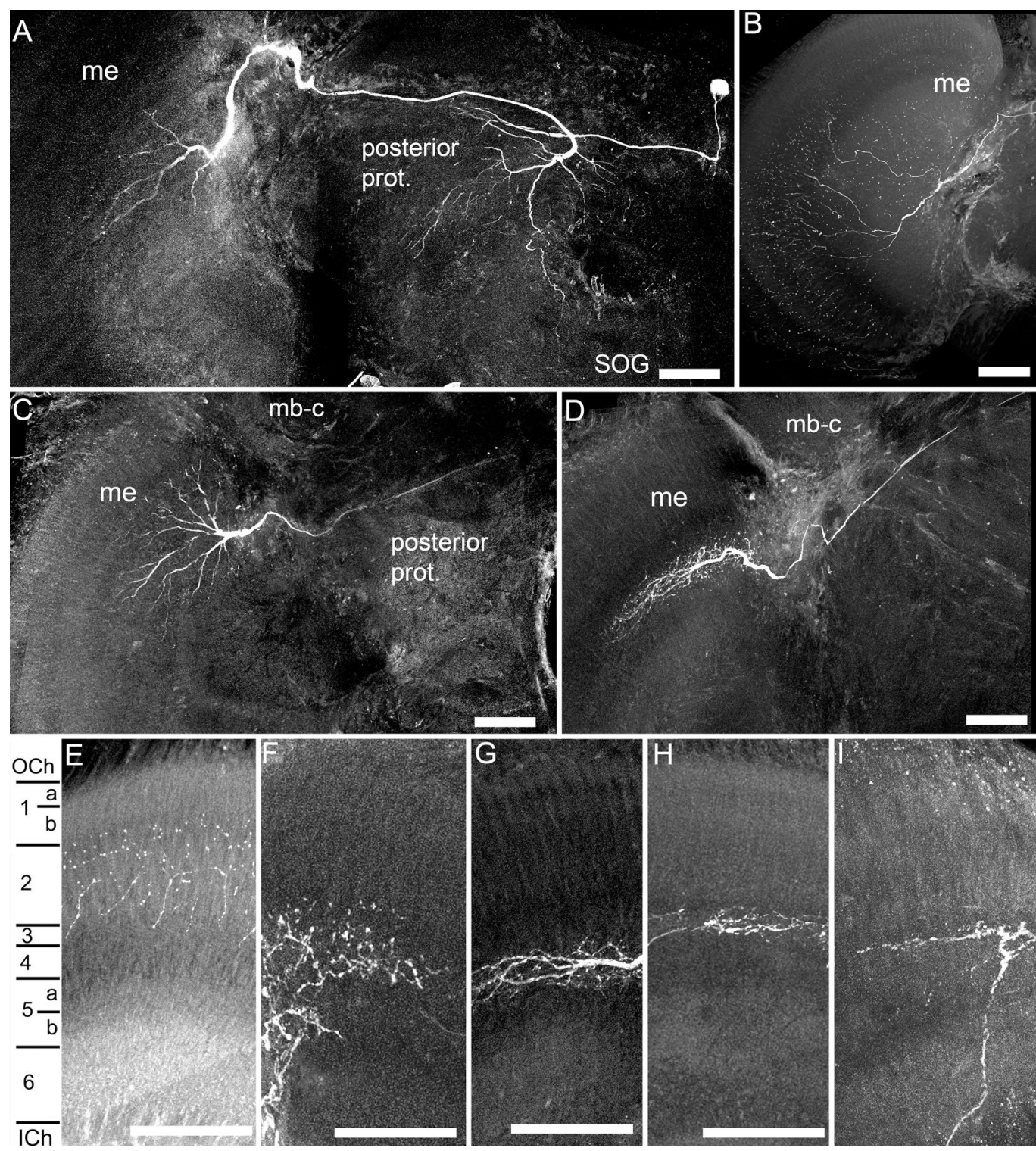

Fig. 2.

Anatomical characteristics of recorded medulla neurons. A. Frontal view of a large field medulla neuron, with branching in the dorsal medulla (me), and which projected posteriorly into the posterior protocerebrum (prot.), with a fine process toward the subesophageal ganglion (SOG). B. A large field medulla neuron, with branching throughout most of the medulla. C. A large field medulla neuron with branching in the dorsal medulla, and a faded projection across the brain in the posterior protocerebrum. D. A large field medulla neuron with branching patterns in the dorsal medulla. E-I. The fine branching patterns in the layers of the medulla, with different neurons entering either the outer medulla layers $(\mathbf{E}, \mathbf{F})$, or the inner medulla layers (G-I). E-G, large field medulla neurons; H,I, amacrine medulla neurons. Scale bars $=100 \mu \mathrm{m}$. 

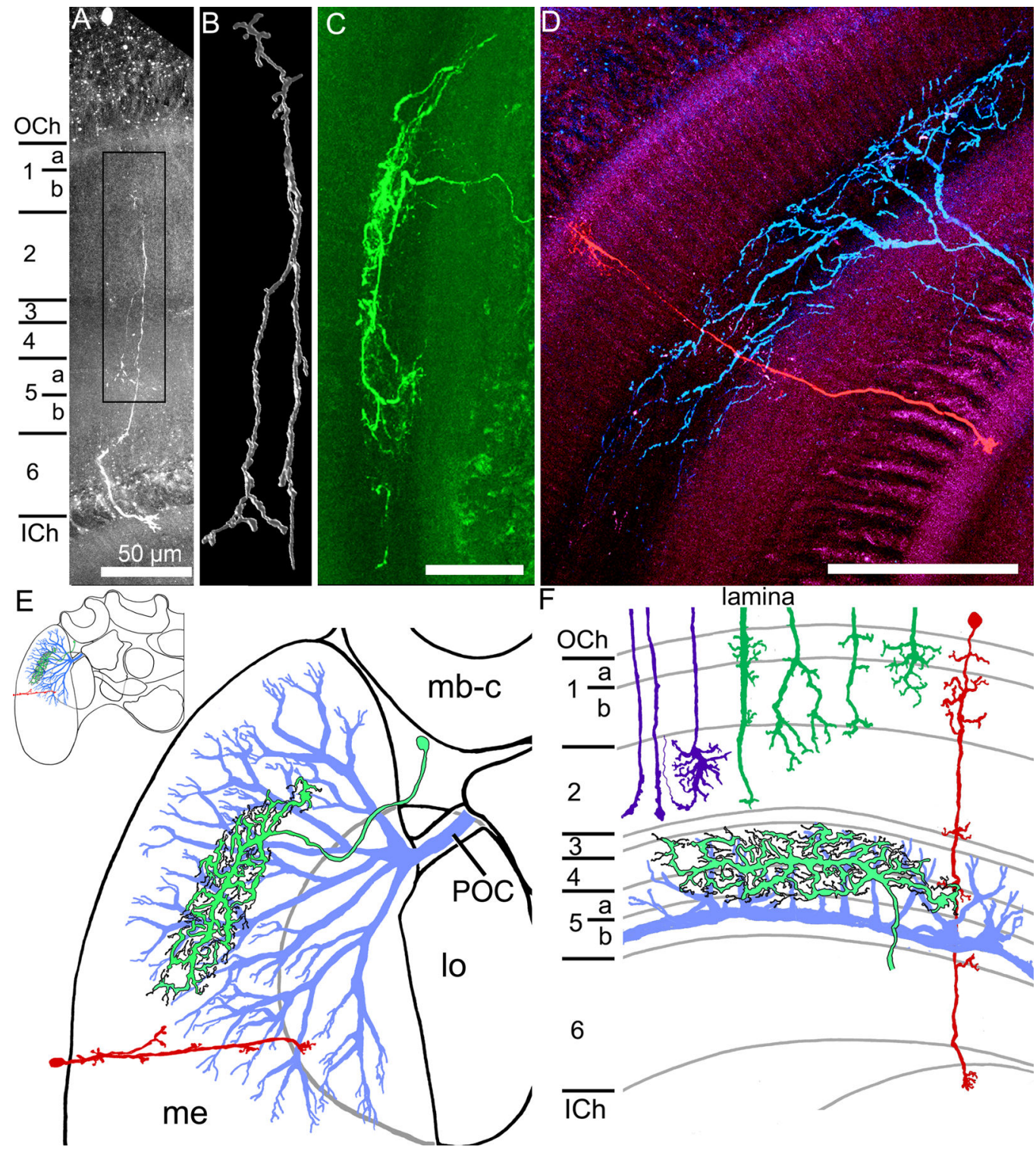

Fig. 3.

Transmedullary, amacrine, and wide field medulla neurons. A. A transmedullary neuron, with a cell body in the outer chiasm, and fine processes in different layers in the medulla, finally projecting into the outer lobula. B. A higher magnification three dimensional reconstruction of the processes in the box in $\mathbf{A}$, with numerous projections and branching along the main neurites. C. An amacrine neuron with branching in the medulla (a cropped view of the processes of this neuron is in Fig. $2 \mathrm{H}$ ). D. Two medulla neurons recorded from and filled in the same brain, with one transmedullary neuron (red) and one large field neuron (blue). E. Schematic diagrams (frontal view)of the three neural types, indicating some general morphological aspects of the large field (blue), amacrine (green with black outline), and transmedullary (red) neurons within the medulla (me), with the transmedullary neuron projecting to the lobula (lo). mb-c: mushroom body calyx; POC: posterior optic commissure; Inset: half of the bumblebee brain $\mathbf{F}$. The same neurons are represented by their relative 
branching patterns in the layers of the medulla, along with the R7-R9 photoreceptor inputs (purple) and the lamina inputs (dark green). Horizontal view; scale bars $=100 \mu \mathrm{m}$ unless otherwise indicated. 


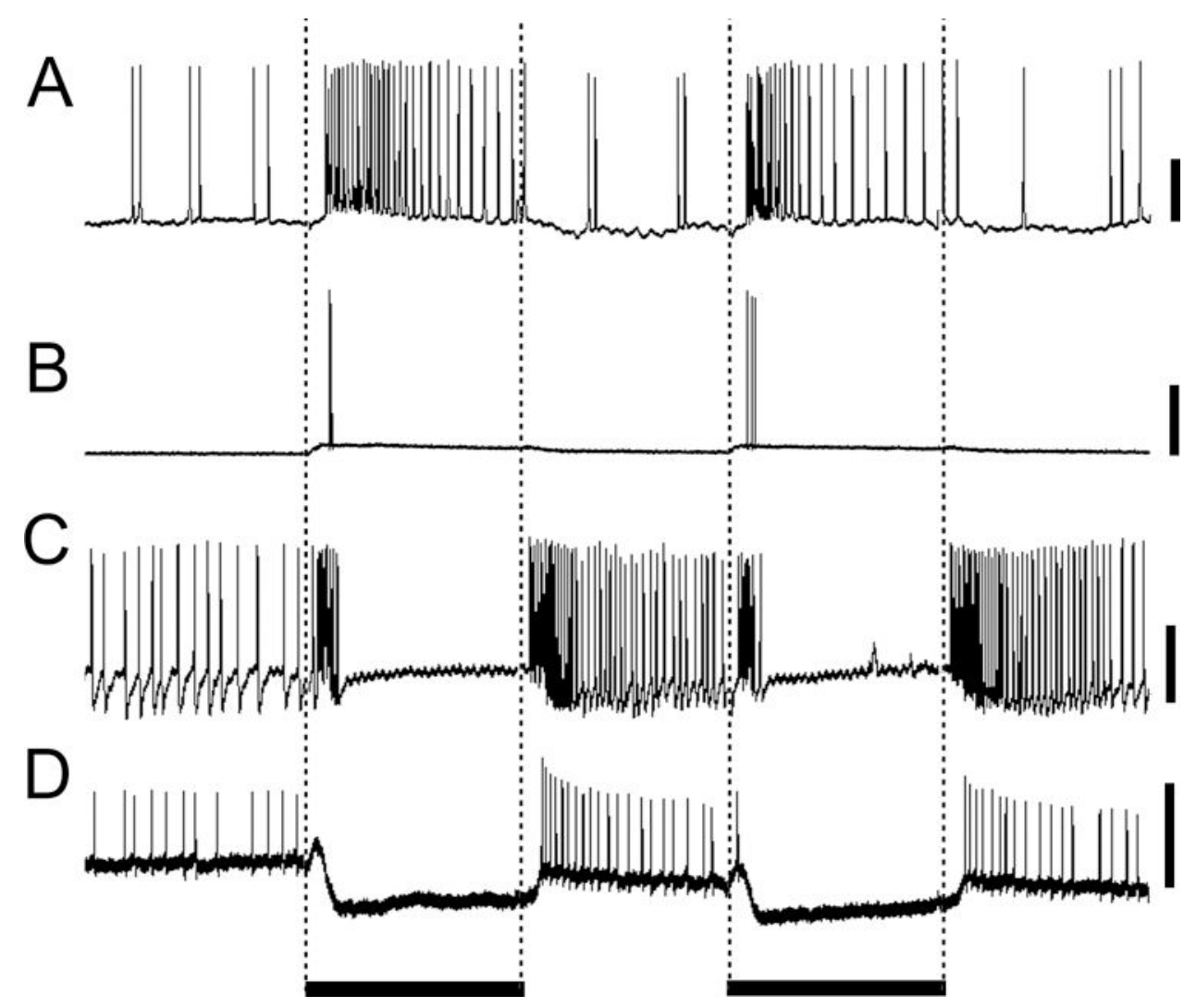

Fig. 4.

Types of medulla neuron temporal response patterns to five $500 \mathrm{~ms}$ light flashes: phasictonic (A, amacrine neuron), phasic (B, large field neuron), on-off ( $\mathbf{C}$, large field neuron), and tonic inhibition with rebound (D, transmedullary neuron). Black bars below the traces signify the duration of the light flashes; scale bars are $20 \mathrm{mV}$. 


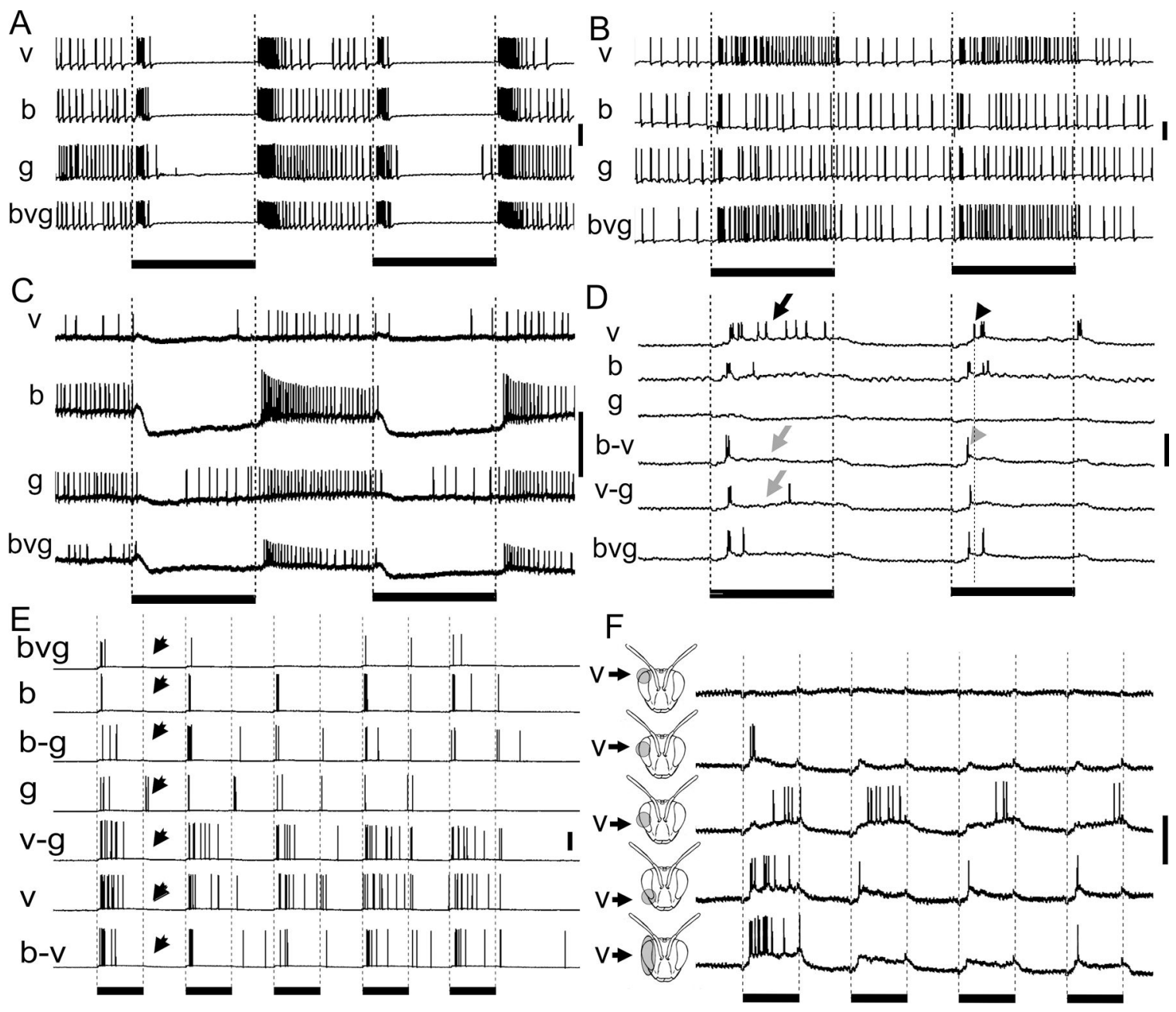

Fig. 5.

Color sensitivity in medulla neurons. A. Broad-band sensitivity. This large field medulla neuron exhibited clear on-off responses to blue (b), green (g), and violet (v) stationary light flashes and to a combination of the three (bgv). B. Narrow-band sensitivity. This large field medulla neuron responded to violet light with a phasic-tonic response, but only had small phasic responses to blue and green light, indicating the neuron may receive only ultraviolet photoreceptor input. C. Narrow-band sensitivity. This transmedullary neuron was strongly inhibited by blue light and only slightly inhibited by violet and green light, indicating that it likely receives blue photoreceptor input. D. Color opponency. This amacrine neuron produced a tonic response to violet (black arrow), a phasic response to blue, and no response to green. When violet was presented along with blue, the cell responded with a shorter latency phasic burst (gray arrow and arrowhead; dotted line indicates the onset of the violet only response), indicating blue inhibited the violet response. Even though the cell had no visible response to green, the violet response was also inhibited by green (grey arrow). The tonic response to violet was present in other trials in this same cell (Fig. 5F). E. Temporal interactions among colors. In this large-field medulla neuron, the cell produced a phasic 'on' response to blue and a phasic-tonic response to violet while it produced a phasic on-off response to green light; the green 'off' response (black arrows) is suppressed by 
simultaneous blue or violet light indicating the color of the stimulus alters the temporal response patterns of the neuron. F Responses of a wide-field neuron with complex receptive field organization. No response to stimulation of the dorsal most part of the eye; stimulation of the dorso-frontal eye results in a short 'on' burst that adapts with repeated stimulation; stimulation of the mid-frontal eye region results in a delayed, more tonic excitation; stimulation of the ventral eye gives rise to a faster inset, tonic response that adapts with repeated stimulation; stimulus extent indicated by grey areas on eye in insets. Scale bars to the left of the traces $=20 \mathrm{mV}$. Black bars under the traces signify the duration of $500 \mathrm{~ms}$ light flashes. 


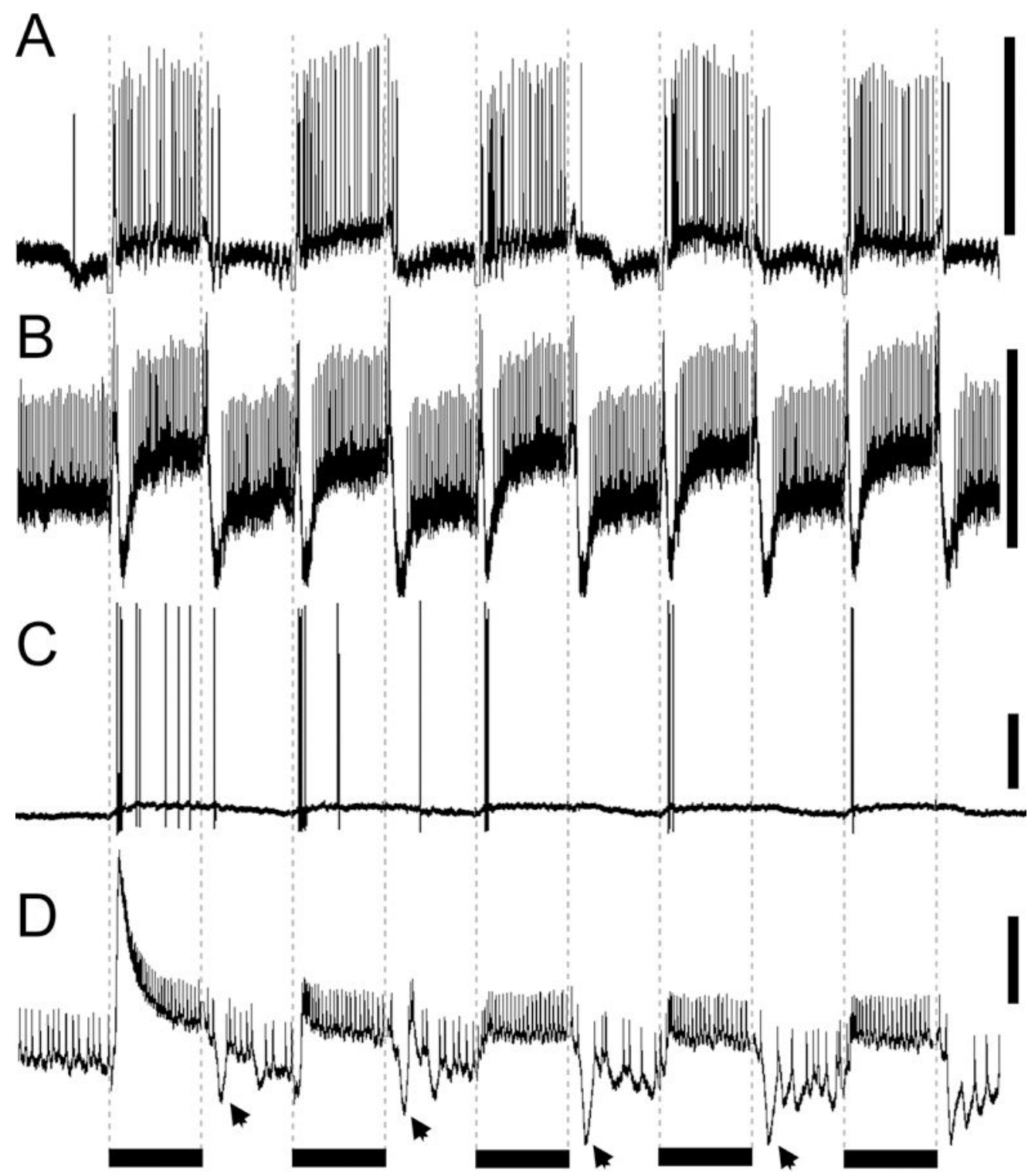

Fig. 6 .

Temporal response properties. A. Large field medulla neuron with tonic response to stationary light flashes. B, Transmedullary neuron with on-off responses. C. Amacrine neuron with strong adaptation after the first stimulus. D. In one large field neuron, the phasic-tonic excitatory response decreased over time while the inhibitory 'off' response (black arrows) increased in amplitude over subsequent stationary light flashes. Scale bars to the left of the traces $=10 \mathrm{mV}$. Black bars under the traces signify the duration of $500 \mathrm{~ms}$ stationary light flashes, which are also indicated by the dotted lines. 


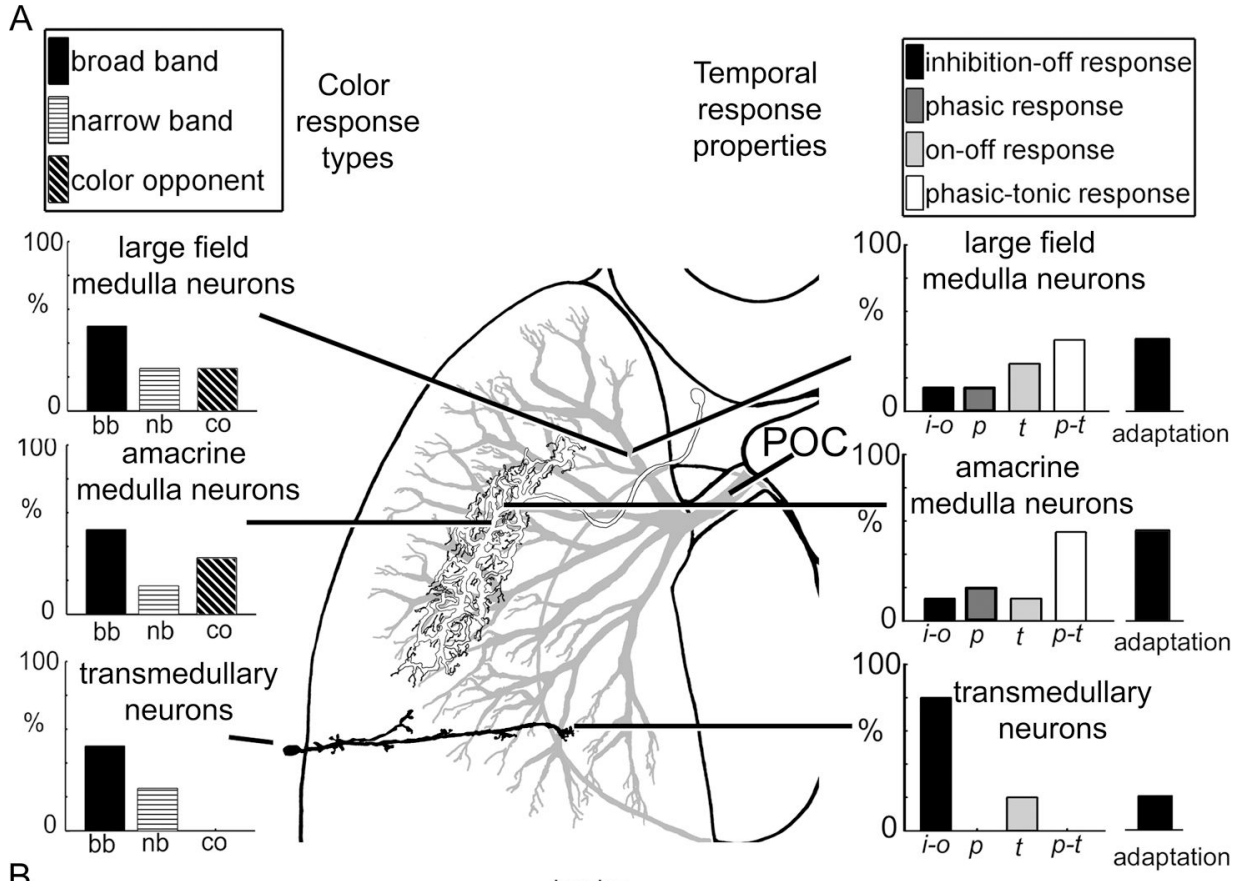

B

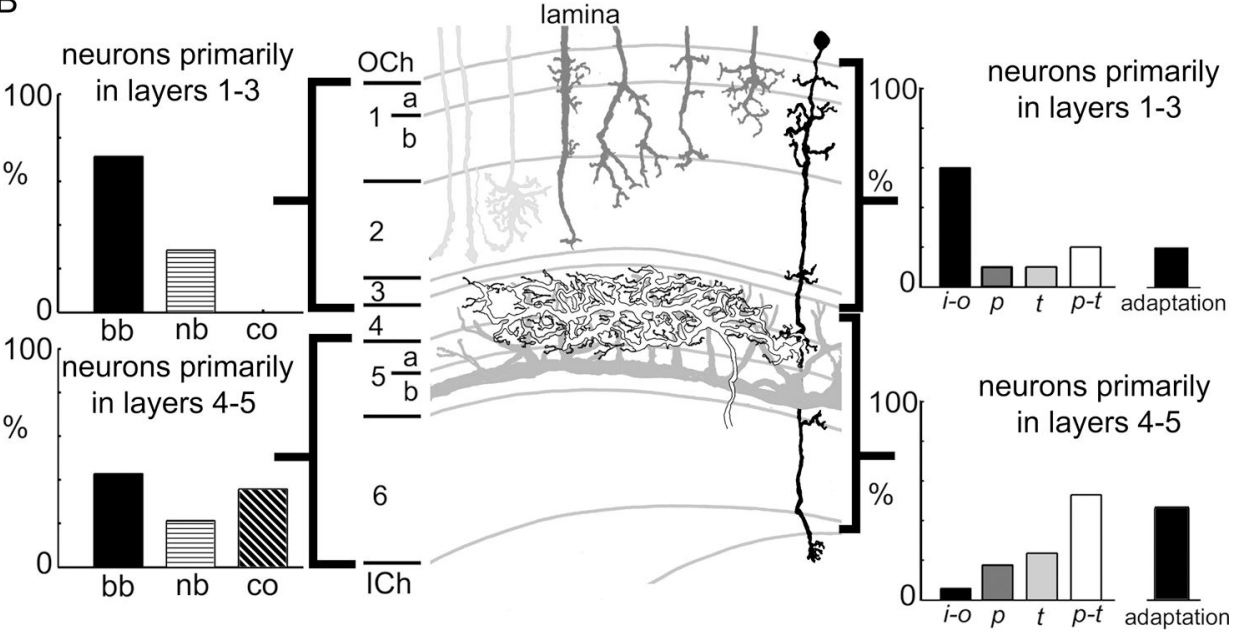

Fig. 7.

Summary of the medulla neurons and their physiological responses to color and to stationary light flashes. A. The distribution of color sensitivity (left) and temporal response characteristics and percent of neurons exhibiting adaptation (right) of the large field (grey), amacrine (white), and transmedullary (black) neurons. The large field medulla neurons output via the posterior optic commissure (POC). B. The distribution of color sensitivity (left) and temporal response characteristics and percent of neurons exhibiting adaptation (right) of neurons branching primarily in layers 1-3 versus those branching in layers 4-5 in the medulla. Photoreceptor input neurons (light grey) and lamina monopolar neurons (dark grey) are represented in the layers as well. 
Table 1.

Information on the immunogen used to label for serotonin, histamine, or GABA in the bumblebee brain.

\begin{tabular}{llc}
\hline Antigen & Immunogen & $\begin{array}{l}\text { Manufacturer, species, type, catalog } \\
\text { number }\end{array}$ \\
\hline Serotonin & $\begin{array}{l}\text { Serotonin coupled to bovine serum albumin (BSA) with } \\
\text { paraformaldehyde }\end{array}$ & Immunostar, Rabbit, Polyclonal, \#20080 \\
Histamine & $\begin{array}{l}\text { Synthetic histamine coupled to succinylated keyhole limpet } \\
\text { hemocyanin (KLH) with carbodiimide (CDI) linker }\end{array}$ & Immunostar, Rabbit, Polyclonal, \#22939 \\
GABA & GABA coupled to BSA & Sigma, Rabbit, Polyclonal, A2052 \\
\hline
\end{tabular}


Table 2.

Anatomy of the recorded medulla neurons. The medulla neurons projected into different layers of the medulla, sent their projections into different areas of the brain (such as the lobula or the protocerebrum), and, when filled, the cell bodies were located in certain areas of the brain (such as lateral to the mushroom body (MB) calyx), which depended on the medulla neuron type.

\begin{tabular}{|c|c|c|c|c|c|c|c|c|c|c|c|c|}
\hline \multirow[t]{2}{*}{ Neural category } & \multicolumn{6}{|c|}{$\begin{array}{l}\text { Number of neurons with neurites in } \\
\text { medulla layers }\end{array}$} & \multicolumn{3}{|c|}{$\begin{array}{l}\text { Number of neurons with projections } \\
\text { into: }\end{array}$} & \multicolumn{3}{|c|}{$\begin{array}{l}\text { Number of neurons with visible } \\
\text { cell body location: }\end{array}$} \\
\hline & 1 & 2 & 3 & 4 & 5 & 6 & $\begin{array}{c}\text { Lobula } \\
\text { layers 1- } \\
\quad 2\end{array}$ & $\begin{array}{l}\text { Contr. } \\
\text { medulla }\end{array}$ & $\begin{array}{l}\text { Posterior } \\
\text { prot. }\end{array}$ & $\begin{array}{l}\text { Lat. to } \\
\text { MB }\end{array}$ & $\begin{array}{l}\text { Med. to } \\
\text { MB }\end{array}$ & $\begin{array}{l}\text { Distal to } \\
\text { the outer } \\
\text { medulla }\end{array}$ \\
\hline $\begin{array}{l}\text { Transmedullary } \\
(\mathrm{n}=5)\end{array}$ & 6 & 4 & 0 & 1 & 1 & 2 & 4 & - & - & 0 & 0 & 3 \\
\hline Amacrine $(n=7)$ & 0 & 1 & 1 & 5 & 2 & 1 & - & - & - & 5 & 0 & 0 \\
\hline $\begin{array}{l}\text { Large field } \\
(\mathrm{n}=15)\end{array}$ & 2 & 3 & 3 & 11 & 11 & 2 & 0 & 4 & 8 & 5 & 3 & 0 \\
\hline
\end{tabular}


Table 3.

Distribution of physiological response properties of the tested neurons among the three types of medulla neurons.

\begin{tabular}{lccc}
\hline \multicolumn{1}{c}{ Physiological response properties } & \multicolumn{2}{c}{ Number of neurons in each medulla category } \\
& Transmedullary $\mathbf{n}=\mathbf{5}$ & Amacrine $\mathbf{n = 7}$ & Large field $\mathbf{n = 1 5}$ \\
\hline Wide receptive field (out of $\times$ tested) & Not tested & $0(1)$ & $5(6)$ \\
Narrow receptive field (out of $\times$ tested) & Not tested & $1(1)$ & $0(6)$ \\
Antagonistic receptive field (out of $\times$ tested) & Not tested & $0(1)$ & $1(6)$ \\
Motion response (out of $\times$ tested) & $1(1)$ & Not tested & $3(6)$ \\
Directional (d) / non directional (nd) (out of $\times$ tested) & 1 d/0nd (1) & Not tested & $1 \mathrm{~d} / 2$ nd (6) \\
Adaptation to stationary light flashes (out of $\times$ tested) & $1(5)$ & $3(7)$ & $8(15)$ \\
Excitatory input from the UV receptor (out of $\times$ tested) & $1(4)$ & $4(5)$ & $12(14)$ \\
Inhibitory input from the UV receptor (out of $\times$ tested) & $2(4)$ & $1(5)$ & $1(14)$ \\
Excitatory input from the blue receptor (out of $\times$ tested) & $1(3)$ & $6(7)$ & $10(13)$ \\
Inhibitory input from the blue receptor (out of $\times$ tested) & $2(3)$ & $1(7)$ & $1(13)$ \\
Excitatory input from the green receptor (out of $\times$ tested) & $1(3)$ & $3(6)$ & $9(12)$ \\
Inhibitory input from the green receptor (out of $\times$ tested) & $1(3)$ & $1(6)$ & $0(12)$ \\
\hline
\end{tabular}

